\title{
WCC 2017-A-106: ANALYSIS OF HEART AND LUNG DOSES IN SYNCHRONOUS BILATERAL BREAST CANCER PATIENTS TREATED WITH BILATERAL BREAST IRRADIATION
}

\author{
B.C.Narasimhulu, Monica Malik Irukulla, Syed Fayaz Ahmed, Deepa M Joseph
}

\section{BACK GROUND:}

Primary synchronous bilateral breast cancer (PSBBC) is a rare clinical entity. The reported incidence ranges between $0.3 \%$ and $12 \%$. PSBBC is defined as having a contralateral tumor diagnosed simultaneously or within 1 year of the initial diagnosis.

\section{MATERIALS AND METHODS:}

Records of patients of PSBBC treated at our institute were reviewed. The demographic data of these patients were retrieved from the case records and dosimetric data from treatment planning system. Data recorded included family history, surgical techniques used, tumor stage and grade, estrogen (ER) and progesterone receptor (PR) status, HER- 2 new expression, neoadjuvant and adjuvant treatment, mean heart and Lung radiation dose.

\section{RESULTS:}

Six patients of PSBBC were treated at our institute between 2015-2016. Median age was 59 years (range 4570 years). All patients were post-menopausal. Five patients had node positive disease of which three patients had perinodal tumor deposits. No significant family history of malignancy was noted. Three patients received neo adjuvant chemotherapy, three received adjuvant chemotherapy. Cumulative doses of Adriamycin given to five patients were documented. No patient received Inj. Trastuzumab. Five patients

Article received on 25 FEB 2017, published on 08 MAR 2017.

B.C.Narasimhulu ${ }^{1}$, Monica Malik Irukulla ${ }^{2}$,Syed Fayaz Ahmed ${ }^{3}$,Deepa M Joseph $^{4}$

${ }^{1}$ Junior Resident, Department of Radiation Oncology, NIMS,India

${ }^{2}$ Associate Professor \&I/C HOD, Department of Radiation Oncology, NIMS, India

${ }^{3}$ Assistant Professor, Department of Radiation Oncology, NIMS, India

${ }^{4}$ Assistant Professor, Department of Radiation Oncology, NIMS, India

Corresponding Author: B.C.Narasimhulu

Email: bcnarasimhulubc@gmail.com
Underwent bilateral modified radical mastectomy, one underwent bilateral breast conservative surgery. Infiltrating duct cell carcinoma was most common histology with 4 patients, Infiltrating duct cell carcinoma with ductal carcinoma in-situ was found in one patient and one had infiltrating tubulo-lobular carcinoma. Out of 12 tumors, 6 tumors were stage IIA, 3 tumors stage IIIA, 2 tumors stage IIIC and one tumor was stage IIB. 5 tumors were Grade III, 5 tumors Grade II, and 2 tumors were Grade I. 9 tumors were ER positive and 3 tumors ER negative. 8 tumors were PR positive, 4 tumors being PR negative. 9 tumors were HER 2neu Negative, 2 tumors equivocal and one tumor HER 2neu positive. For all patients, treatment planning and dose calculation were done on CT simulation images using ECLIPSE planning system. The dose of 40Gy in 15 fractions was given to the bilateral chest wall and bilateral supraclavicular fossa with 3DCRT technique on Linac. One patient received dose of 50Gy to the bilateral breast and left supraclavicular fossa. Mean Heart dose range from 3-3.48cGy and Mean V15 of left Lung ranged from $19-32.84 \%$. For one patient treated with to 50Gy, the mean heart dose was 6.23Gy and Mean V20 of Lung was $25.06 \%$ all patients completed treatment and no patient had grade III toxicity during treatment and follow up. Currently, five patients are on endocrine therapy.

\section{CONCLUSION:}

Bilateral breast irradiation is technically challenging. Dose to the Heart and Lungs needs to be minimized to decrease the long term toxicity which is possible with current techniques (3DCRT, IMRT, IGRT and Tom therapy). In our series of patients, simultaneous bilateral chest wall irradiation and regional nodal irradiation could be done while maintaining the acceptable dose limits to the Heart and Lungs. 